\title{
Foresight Analysis at the Regional Level - A Participatory Methodological Framework
}

\author{
Anastasia Stratigea ${ }^{1} \&$ Chrysaida - Aliki Papadopoulou ${ }^{1}$ \\ ${ }^{1}$ School of Rural and Surveying Engineering, National Technical University of Athens, Athens, Greece \\ Correspondence: Anastasia Stratigea, School of Rural and Surveying Engineering, National Technical University of \\ Athens, Heroon Polytechniou str. 9, Zographou Campus, Athens 15789, Greece. Tel: 30-210-772-2672. E-mail: \\ stratige@central.ntua.gr
}

Received: February 19, 2013

Accepted: March 12, 2013

Online Published: April 27, 2013

doi:10.5430/jms.v4n2p1

URL: http://dx.doi.org/10.5430/jms.v4n2p1

\begin{abstract}
The focus of the present paper is on the potential of participatory scenario planning as a tool for regional future studies. More specifically, a methodological framework for participatory scenario planning is presented, integrating an analytical participatory scenario planning approach (the LIPSOR model) with the Focus Groups and Future Workshop participatory tools. This framework is applied to a Greek rural region, for building scenarios and structuring policies for its future rural development. The specific region is in front of a great challenge referring to the potential location of a large infrastructure (airport), which can drive a considerable socio-economic restructuring, affecting mostly the agricultural sector due to the land loss and the pressures exerted from competitive land uses around the airport area.
\end{abstract}

Keywords: participatory planning, scenario planning, LIPSOR model, focus groups, future workshop, rural regions, policy

\section{Introduction}

Rural regions, accounting for a large part at the European but also the global scale, are nowadays in front of great challenges as to their future sustainable development. Most of them are confronting problems of decline and out-migration, ageing of their population, a lower skill base and average labor productivity, etc. (Organisation for Economic Co-operation and Development [OECD], 2006), which are strongly affecting their future development perspectives. Moreover, they have to deal with new challenges emerging from the external environment, such as climate change, changing consumption patterns, technological developments, increasing urbanization patterns, etc.

In order to effectively cope with challenges of the internal and external environment, a society empowering, place-based, cross-sectoral approach for policy making needs to be adopted, pursuing the sustainable exploitation of available resources and human capital, as well as the better coordination and interaction among sectors, levels of government and public and private actors (Stratigea, 2011). Towards this end, planners are challenged to focus on the development of tools and approaches that will support policy makers in making knowledgeable decisions towards desirable futures that can be well adjusted to the peculiarities of each specific rural context. The focus of the planning efforts, in such a context, is not only on the policy results delivered, but also on the process through which these results are produced and the way tools used can structure thoughts and support an effective communication platform for interaction among local participants in a planning exercise. Such an effort implies the need for planning tools that can handle uncertainty and complexity and support a more pluralistic approach (Godet, 1994), capable of integrating information on local views, opinions, visions etc. in the decision making process.

Along these lines, the focus of the present paper is on the structuring of a participatory methodological framework, for planning the integrated future agricultural development of a specific Greek rural region, the region of Kastelli-Herakleion in Crete. This framework is based on the LIPSOR participatory analytical model, integrated with the Focus Groups and the Future Workshop participatory tools. The structure of the paper has as follows: Section 2 refers to the problem to be dealt with in the specific study region; in Section 3 the participatory methodological framework is discussed; in Section 4, the empirical results of the application of this framework in the region at hand are presented; and finally, in Section 5, some conclusions are drawn. 


\section{The Problem}

A critical issue for the long term survival/flourishing of the agricultural sector in rural regions is associated with the pressure exerted on agricultural land from other economic sectors (e.g. tourism), but also from the expanding urbanization patterns, the deployment of large scale infrastructure works etc., affecting the land use pattern in rural regions.

The Kastelli case study, a traditionally agricultural area of the Crete region (municipality of Kastelli - Herakleion), is confronting such a challenge, emerging from the potential location of a large infrastructure in the area (a new airport). This situation, apart from the environmental impacts, will also place a large burden on the social and economic structure of the region, mainly due to: the loss of fertile agricultural land that will be taken away for the airport infrastructure; and the pressure expected on agricultural land from other sectors, seeking for a location around the airport area, thus implying a certain restructuring of land uses and local economic profile.

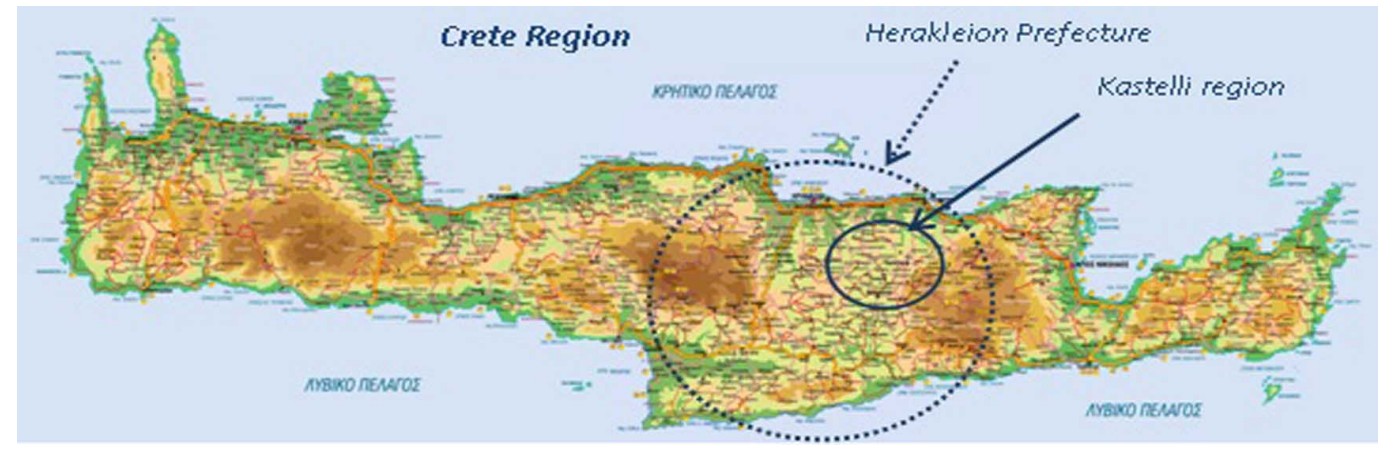

Figure 1. The location of the Kastelli municipality

The Kastelli municipality is a rural area, located at the eastern part of the Herakleion Prefecture - Crete Region (Figure 1). The area consists of a mountainous and a flat part, the fertile Kastelli plain that largely contributes to the agricultural production of the whole Herakleion Prefecture.

The area of Kastelli is about 12332 hectares, with 6819 inhabitants (2001). The population is distributed at the fourteen municipality settlements, while a quarter of the population lives in the Kastelli settlement, being the economic, administrative and service center of the municipality. In the local economic structure, the agricultural sector prevails, while manufacturing has a low presence. The same holds for the tourist sector, despite the remarkable natural and cultural resources available in the region. More than one third of the working population is occupied in the primary sector, a fact that is mainly due to the fertile soil of the Kastelli plain, which fits quite well with the development of this sector.

In dealing with the challenge of the potential location of the airport, an 'integrated agricultural development plan' for the Kastelli region was developed, aiming to explore plausible future developments of the region under the new circumstances. The objectives pursued by this plan have as follows:

- Environmental protection: seeking for the rational exploitation of local natural resources;

- Social cohesion: ensuring equal access of local population to job and income opportunities, health and education services, etc.;

- Economic efficiency: placing emphasis on the adoption - use of technological innovations for increasing efficiency in the agri-food and other economic sectors;

- Agricultural development: strengthening the development of the agricultural sector that, based on the 'multifunctionality' potential of the agricultural land, can form the 'vehicle' for the flourishing of the local productive system (agri-food production, manufacturing, agrotourism, energy production, etc.);

- Energy production: exploiting the renewable energy production potential of the region (agri-waste management, energy crops, solar and wind energy, etc.);

- Accessibility: upgrading of the road transport network connections;

- Innovation diffusion: increasing productivity and competitiveness of the local productive system; and

- Preservation of cultural heritage: pursuing the rational exploitation of the cultural heritage of the Kastelli region. 
For the development of this plan, a participatory approach was adopted, aiming at strengthening the interaction among planners, policy makers, local municipality authorities, stakeholders and local community in the planning process. In the following, the participatory methodological approach, structured for this purpose, is presented.

\section{The Proposed Participatory Methodological Framework}

The Kastelli scenario planning exercise integrates the LIPSOR analytical participatory approach, being the main participatory tool of the proposed framework, with the Focus Groups methodology and the Future Workshop participatory approach. This framework aims at structuring and evaluating scenarios for the future integrated agricultural development of the Kastelli region, taking also into account the perspective of the new airport location. The whole process is conducted in three consecutive stages that have as follows (Figure 2):

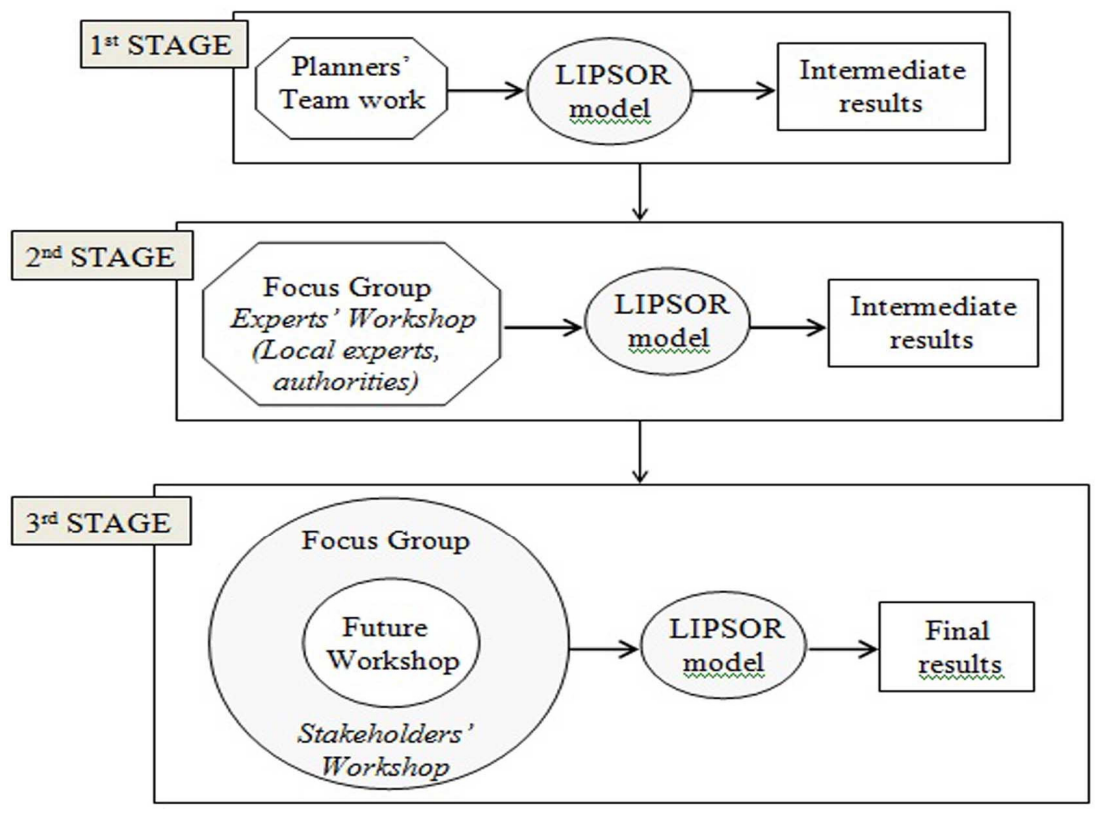

Figure 2. The proposed participatory methodological framework

Source: Stratigea, Giaoutzi \& Papadopoulou, 2010.

- $1^{\text {st }}$ stage: relates to the planners' work, consisting of the: gathering of various forms of data, relating to the study region; further elaboration of these data to create the necessary input that feeds the LIPSOR model; first round of intermediate results from this model, which formed the basis for running into the second step.

- $2^{\text {nd }}$ stage: first round results were discussed with local authorities and experts in a workshop. Input, views, comments etc. gathered in this workshop drove the refinement of data input, reflecting local expertise and knowledge. The refined input feeds the LIPSOR model, producing a second round of intermediate results, which provided the basis for turning into the third step.

- $3^{\text {rd }}$ stage: in this stage, a stakeholders' workshop was organized, in which results obtained by the second round were presented and validated on the basis of the stakeholders' views. Eight participants were selected in this respect, representing different stakes of the local community. The structure of the stakeholders' workshop was based on the focus groups methodology. The running of the focus group discussion was based on the future workshop participatory context, structuring the discussion among stakeholders in a set of successive steps. Within these steps, participants had the chance to identify local problems, vision desirable future developments and discuss policies towards these developments. Within the Focus Group discussion, the context of the future workshop structure evoked a rich and fruitful interaction among stakeholders, which added value to the whole effort, by providing information on local expectations, visions for the future of their region, policies relevant to reach desired futures that can be well adjusted to the local peculiarities, etc. The outcome of the workshop was a further enrichment of the input data collected so far, which were used for a final round of the LIPSOR analytical model, placing thus the final results in the specific local context.

A short description of the three participatory tools is provided below, before presenting the results obtained by the 
application of the above methodological framework in the Kastelli case study.

\subsection{The LIPSOR Analytical Participatory Framework}

The LIPSOR framework consists of five discrete parts-modules (Godet, 1994 and 1999):

- The MICMAC module: explores the key drivers of change in a system and formulates the basic questions as to its future states. It is based on a structural analysis, depicting the influence-dependence relationships among key drivers of both the internal and the external environment that can steer the future states of the system.

- The MACTOR module: identifies the role of key actors of a system by studying their attributes and the influence-dependence relationships among them that can reveal underlying power relationships or potential alliances. Moreover, it explores the attitude (level of resistance) of the various actors against the objectives of a planning exercise, providing planners with valuable information for policy purposes.

- The SMIC PROB-EXPERT module: consists of an expert-based approach that provides information/opinions on a range of hypotheses, relating to the future development of a study system. The module adopts an explorative approach, based on qualitative information. Simple and conditional probabilities of the above hypotheses are attached by experts in an effort to foresee the future states of the study system as combinations of the above hypotheses.

- The MORPHOL module: is used for a systematic exploration of all possible future developments of the system (the morphological space), structured on the basis of all combinations of possible future states of the key drivers of change involved. Under certain conditions, it can lead to a set of 'plausible scenarios' for the system at hand.

- The MULTIPOL module: is used for the evaluation of the scenarios structured by the previous modules, attempting at the same time to define strategic directions (policies) and actions (measures) for the implementation of each scenario outcome.

Each of the above modules, when used independently, can lead to the formulation of certain scenarios, which differ as to the insight gained, the systematics as well as the range of potential future states reached. Moreover, the five LIPSOR modules can be used in a sequence, leading to a more deep insight into the system at hand and a more elaborated output.

\subsection{The Focus Groups Methodology}

The Focus Groups methodology is a promising scientific tool and a suitable social setting for organizing a social debate (Kasemir et al., 2000). It is also a promising participatory tool for arriving at policy-oriented assessments. It may serve as a platform for social learning that brings together scientific knowledge and behavioural patterns of citizens.

Focus Groups can be described as guided group discussions that are focused on specific topics. The whole process is characterized by its dynamic nature and synergetic effects, resulting in the generation of far more information than in other research methods (Berg, 1989; Stewart \& Shamdasani, 1990). It can deal with complex issues, using knowledge from various scientific disciplines and/or stakeholders, so that integrated insights are made available to decision makers (Rotmans, 1998). The whole process is divided into three steps:

- Step 1 refers to the 'planning' of the whole exercise, including decisions such as: number of sessions and time devoted to each session; selection of participants; planning of the discussion by creating an interview guide; preparation of the material to be presented to the participants; etc.

- Step 2 refers to the 'running' of the whole exercise, on the basis of the predefined interview guide. The whole process starts with the presentation of the informative material, designed to introduce the issue and motivate participants' discussion. Then interaction among participants is encouraged, for gathering different views/opinions on a well structured set of questions.

- Step 3 refers to the 'elaboration of qualitative results' and the production of the final report. Various qualitative analysis tools can be useful in this respect (see Stratigea, Grammatikogiannis \& Giaoutzi, 2012).

Based on deliberately presented inputs and specific rules, Focus Groups can be considered as social experiments, capable of: producing collective judgments, revealing communication barriers, studying conflict behaviour, acquiring local knowledge, creating acceptable options, synthesizing information, etc. In such a context, their role is more to increase insights than to produce generalized results (Dürrenberg et al., 1997).

\subsection{The Future Workshop Approach}

The Future Workshop participatory approach aims at involving people in creating their own preferred future, 
strengthening thus the democratization of the decision making process in planning and policy making. The Future Workshop approach seems also to be a very promising tool for generating knowledge from interactive learning and facilitating creativity among participants (Jungk \& Mullert, 1987). Future Workshop is considered as a: participatory tool supporting public involvement in a dialogue process, which provides more insight into the problem/region at hand and improves the decision making process; learning tool for all parties involved, i.e. planners, decision makers and the participants themselves; tool supporting creativity and generation of new ideas in the process of exploring future developments of a problem/region at hand.

The goals pursued in the context of the Future Workshop approach are:

- to get insight into the type and diversity of problems for a certain study region/issue, as these are perceived by participants;

- to create shared visions as to desired future developments;

- to define strategies towards the achievement of a desired future; and

- to achieve mutual understanding among participants, as a prerequisite for building consensus towards the identification and prioritization of problems.

Participants in a Future Workshop are expressing their views in the context of a well structured discussion, which takes place along four discrete steps (Barbanente, Khakee \& Puglisi, 2002):

- $1^{\text {st }}$ step - Preparation phase: at this step, the preparation of the Future Workshop is taking place, involving decisions on the issue to be discussed, the structure of the discussion, the material to be presented, the place of sessions, etc. Participants are also selected on the basis of certain criteria. After the accomplishment of the above preparatory steps, the discussion starts with the presentation of goal and objectives of the planning exercise, while other kind of supportive material is also used for further explanation of issues involved and stimulation of participants' fantasy.

- $2^{\text {nd }}$ step - Critique phase: within this step, negative experiences and dissatisfaction of participants from the current situation are discussed. Data collected are classified into specific thematic fields. Then participants discuss on priorities of these fields, setting, by some system of voting, the issues of greatest importance, according to their views. Information collected at this stage helps the planner to identify, classify and prioritize the problems of the study area as well as their spatial differentiation.

- $3^{\text {rd }}$ step - Fantasy phase: at this step, a collective brainstorming of participants is motivated. Participants are invited to 'dream' of the future and express their views on possible and desirable future developments, relating to the objectives set. Ideas, visions, fantasies etc. identified at this stage are again grouped by participants into key themes, which are further rearranged in order to end up with alternative coherent visions.

- $4^{\text {th }}$ step - Implementation phase: at the final step, the feasibility of ideas generated in the fantasy phase is considered, by identifying obstacles and restrictions towards their implementation, as well as possible strategies to overcome them.

\section{Application of the Methodological Framework in the Kastelli Region}

The focus of the present section is on the application of the previously described methodological framework in the Municipality of Kastelli - Herakleion Crete.

\subsection{The MICMAC Approach - Identification of Key Variables}

At this stage, a structural analysis for the Kastelli region was carried out. The analysis adopts a systemic approach and provides the ground for an in depth exploration of the region. It is based on a structural table, incorporating 63 variables, which relate to aspects of both the internal and the external environment (Table 1). The scope of the analysis is to provide insight into the regional system at hand, by elaborating on the influence - dependence relationships among internal and external variables of the system. The output of this process is the identification of the 'key drivers of change' of the regional system (Godet, 2001).

The structural analysis has revealed the highly influential role on the evolution of the system of a number of variables, such as the: educational level and skills of local population; research and development; adoption and use of technology / innovation; telecommunication infrastructure and networking; European policy and relating funding in the agricultural, industrial, energy and tourist sector; alternative tourism; etc. 
Table 1. Aspects of the internal - external environment used in the structural analysis of the Kastelli region

\begin{tabular}{|c|c|}
\hline \multicolumn{2}{|c|}{ The Study System: Internal and External Environment } \\
\hline Internal Environment & $\begin{array}{ll}- & \text { environmental aspects } \\
- & \text { population structure } \\
- & \text { local economic structure (primary, } \\
& \text { secondary, tertiary sector) } \\
- & \text { inter/intra regional infrastructure networks } \\
- & \text { technology } \\
- & \text { telecommunication infrastructure / ICTs }\end{array}$ \\
\hline External Environment & $\begin{array}{ll}- & \text { private investments in the various sectors } \\
- & \text { European funding in the various sectors } \\
- & \text { international tourist patterns } \\
- & \text { food quality and safety - new food } \\
\text { consumption patterns }\end{array}$ \\
\hline
\end{tabular}

On the other hand, the 'dependent variables' of the system are identified, i.e. those which are supposed to be influenced by a change in the key variables. Among these are the: pollution of water resources, biodiversity and landscape; local income; traditional and organic agricultural and livestock crops; products of designated origin; abandonment of agricultural land; employment in primary sector; export trade; etc.

The knowledge gained by the application of the MICMAC module, was proved quite useful for structuring the necessary data input for running the rest of the LIPSOR modules.

\subsection{Actors' Games - The MACTOR Approach}

The MACTOR module is focusing on the study of the 'actors' games', seeking to gauge the balance of power among actors and study their convergences or divergences with a certain number of associated stakes and objectives (Godet, Monti, Meunier \& Roubelat, 2004). The scope of the analysis is both: to get insights into the power relationships and potential alliances among actors, by studying the influence - dependence patterns among them; and to explore their position as to the objectives pursued in the study area.

In the Kastelli case study, the actors were selected on the basis of their influence on the key drivers of change, identified in the MICMAC application. They are: A1 - government; A2 - local governmental agencies; A3 - farmers; A4 - environmental agencies; A5 - road transport companies; A6 - marine transport companies; A7 - air transport companies; A8 - tourist firms; and A9 - European Union.

The influence-dependence relationships among actors and the convergence-divergence between actors and the objectives of the study are described by two cross-impact matrices. Elaboration of data incorporated in these matrices by use of the MACTOR module provides:

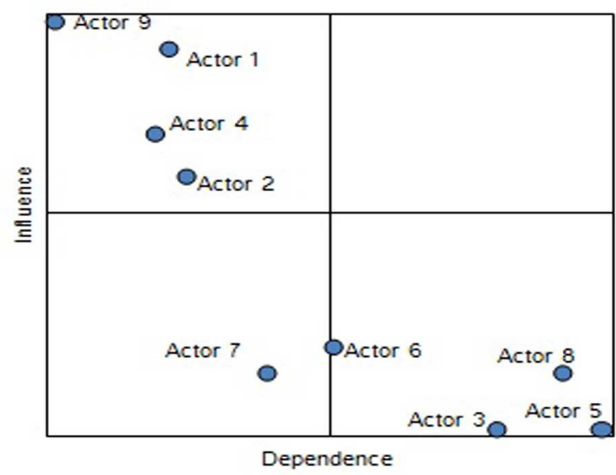

Figure 3. Map of influence - dependence among actors

Source: Stratigea, Giaoutzi \& Papadopoulou, 2010

- An overview of the most 'influential actors' (high influence and low dependence - see Figure 3) for the Kastelli case study, being the: A1 - government, A2 - local governmental agencies, A4 - environmental agencies and A9 European Union; and an overview of the most 'dependent actors' (low influence and high dependence) being the: A3 - farmers, A5 - road transport companies, A6 - marine transport companies and A8 - tourist firms (Figure 3). 
- Indicative results in respect to the position of actors as to the objectives set in the Kastelli region. In Figure 4, indicative results of the convergence - divergence of actors in respect to the objective 'renewable energy production' is presented, where it is noticed that almost all actors agree towards the specific objective, apart from A8 (tourist firms), as the tourist sector is considered to be negatively influenced by the deployment of renewable energy infrastructure. Moreover, a certain convergence appears among almost all actors as to the objectives 'economic efficiency' and 'accessibility', with actor A4 (environmental agencies) being the only opposing actor, for environmental protection reasons; while all actors converge as to the objectives 'innovation diffusion' and 'social cohesion'. Also, a certain divergence among actors is noticed in respect to the objective 'environmental protection', where five actors are opposing, namely the: A3 (farmers), A5 (road transport companies), A6 (marine transport companies), A7 (air transport companies) and A8 (tourist firms). Finally, A8 (tourist firms) is the only actor diverging from the objective 'agricultural development', a fact that implies the competition among the two sectors for land; while all actors converge towards the objective 'preservation of cultural heritage', with the exception of A5 (road transport companies).

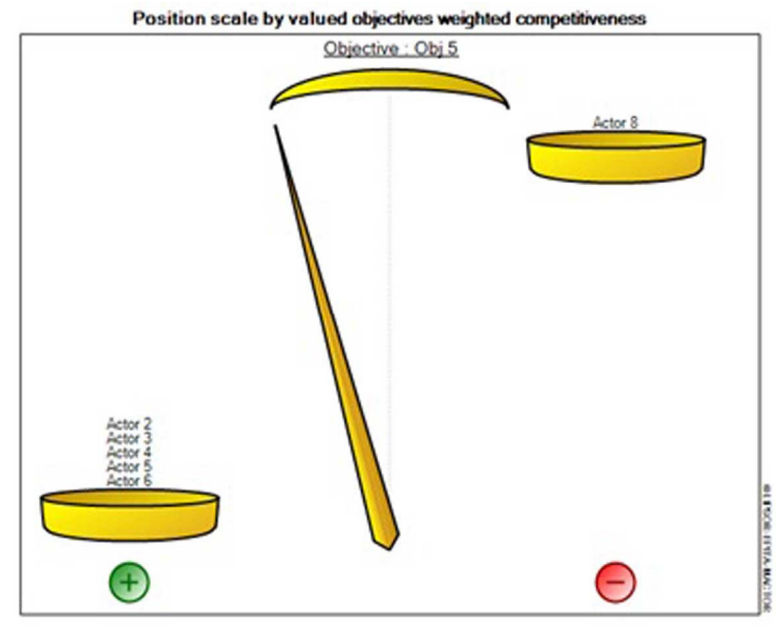

Figure 4. Position of actors as to the objective 'renewable energy production'

Source: Stratigea, Giaoutzi \& Papadopoulou, 2010

\subsection{The Experts' Cross-Impact Analysis - The SMIC PROB-EXPERT Approach}

The goal of this step is to define single and conditional probabilities of certain hypotheses as well as probable future scenarios of the study region on the basis of different combinations of the hypotheses. The steps followed are: first, formulation of five to six basic hypotheses; second, definition of 'simple (Note 1)' and 'conditional (Note 2)' probabilities of these hypotheses by experts; third, proper combination of hypotheses for structuring 'probable scenarios' and ranking of them according to the defined probabilities.

The following hypotheses were set in the Kastelli region, for discussion with the experts:

- H1. 'Multifunctionality of agricultural land serves the goal of integrated development'. Multifunctionality of agricultural land supports the development of a range of activities in the area of concern, which can strengthen the local economic and social profile.

- H2. 'Technology - The adoption of technology can positively affect the various sectors'. For example, in the agricultural sector, adoption of technology can contribute to the increase of agricultural production, the qualitative improvement of agri-food products and the strengthening of competitiveness of the agri-food sector.

- H3. 'Vertical structure of production'. It contributes to the region's economic efficiency.

- H4. 'Information and Communication Technologies (ICTs)'. ICTs can support the diffusion of knowledge, contributing to the upgrading of local skills and the increase of knowledge stock, while they may strengthen the interaction among sectors, serving thus the goal of integrated rural development.

- H5. 'Energy'. Agricultural sector is strongly involved in energy production (processing of energy crops and biomass). 
- H6. 'Location of the new airport in the Kastelli region'.

For the above six hypotheses, simple and conditional probabilities are defined by a group of experts. This information is feeding the SMIC PROB-EXPERT module, providing a set of 'probable scenarios' for the Kastelli region, together with their probabilities to happen. Based on their probability rates, 26 most probable scenarios are selected, which are further classified into two groups (Table 2):

Table 2. Results from SMIC PROB-EXPERT module

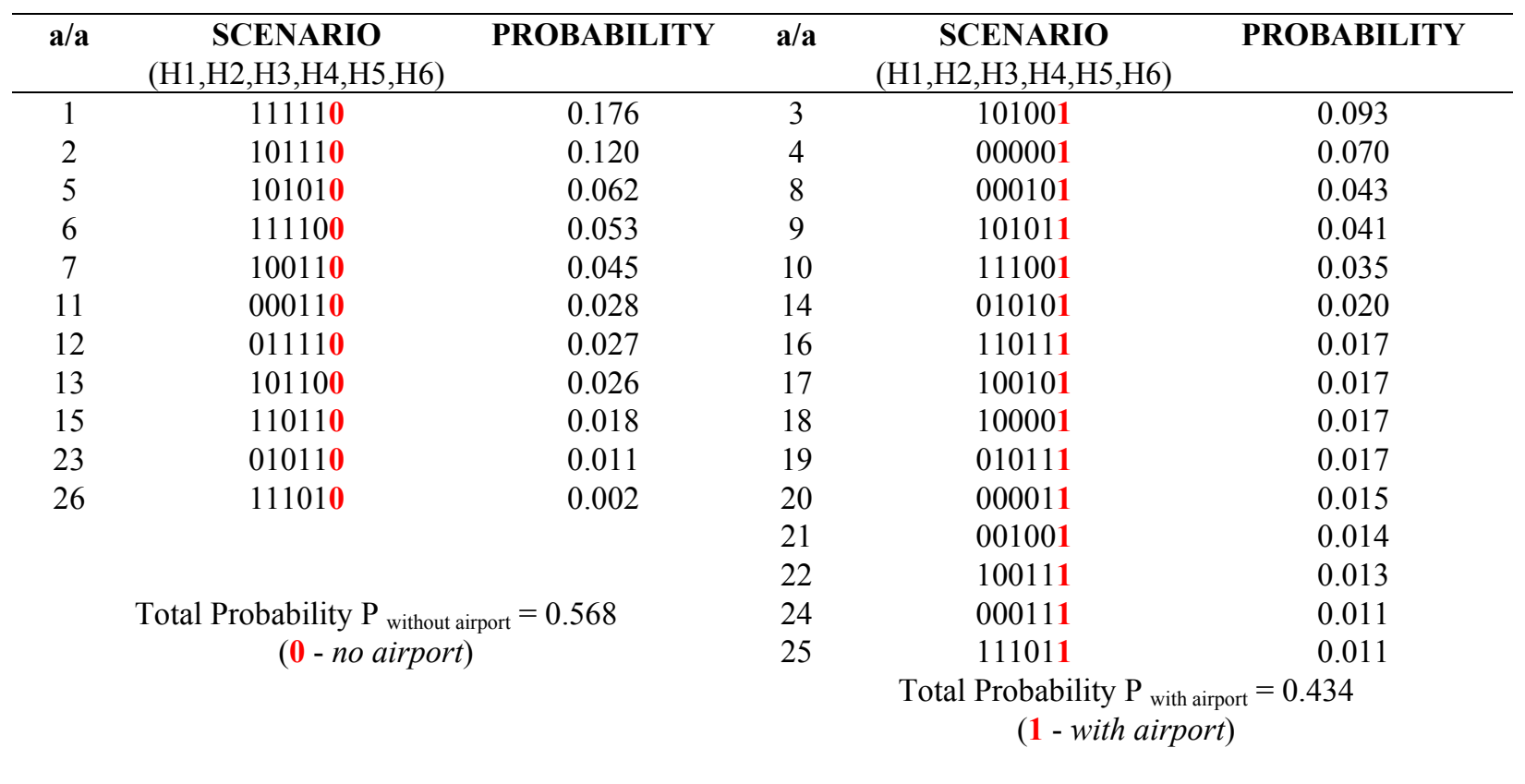

Source: Stratigea, Giaoutzi \& Papadopoulou, 2010

- The first group incorporates those scenarios that do not consider the location of the airport in the Kastelli area (H6=0); and

- The second group incorporates those scenarios that consider the location of the airport $(\mathrm{H} 6=1)$.

The scope of such a classification is the further elaboration of scenarios and the identification of types of hypotheses that are presented in scenarios in a more systematic way. The hypotheses that constantly appear to most of the first group of scenarios (scenarios 'with no airport'), are (Table 2):

- H1: Multifunctionality of agricultural land;

- H4: Information and Communication Technologies (ICTs); and

- H5: Energy

The scenario building process can be mainly based upon this set of hypotheses, in case that the airport will not finally be located in the Kastelli area.

On the contrary, in the second group (scenarios 'with airport') (Table 2), there are no hypotheses that seem to have a more systematic presence in certain scenarios. This can be explained in two ways:

- The construction of the airport may cause a kind of 'destabilization' of the area of concern.

- The construction of the airport may contribute to the region's development through several directions ('open' future).

The knowledge gained through the SMIC PROB-EXPERT module is important for the next step (MORPHOL module), in order to set hypotheses in the scenario building process for both 'with airport' and 'with no airport' future possible developments of the Kastelli region.

\subsection{Scenario Building Process - The MORPHOL Approach}

By combining goals and objectives of the Kastelli case study and the knowledge gained by the previous LIPSOR modules, certain hypotheses were formulated in respect to the future development of the study region, based on the 
following characteristics (variables) of the system at hand:

- 'Settlement patterns of population': this will depend on the final decision for the location of the new airport in the study region. As possible future outcomes of this hypothesis are considered: a) settlement patterns remain unchanged in case that the airport is finally not located in the region; b) a new settlement pattern is emerging in case that the new airport is located in the Kastelli region.

- 'Agri-food technology': refers to the diffusion of technological innovations in the agri-food sector. Possible future developments can range from: a) low, b) medium to c) high innovation diffusion.

- 'Multifunctionality': refers to the multifunctional role of agricultural land. Possible future developments range from: a) low level, b) medium level to c) high level of multifunctionality of agricultural land.

- 'Energy (renewable energy - RE)': the agricultural sector can significantly contribute to the energy production pattern through processing of biomass, derived either from the sector's management of waste or from energy crops. Possible future developments can range from: a) low, b) medium to c) high level of RE share to the energy consumption pattern in the area of concern.

- 'Information and Communication Technologies - ICTs': refers to the level of exploitation of ICTs in the study region. Possible future developments can range from: a) low to b) high level of ICTs exploitation.

- 'Creation of Technological Park': refers to the contribution of a technological park in the development potential of the area of concern. The hypotheses set in this case are: a) low contribution, b) high contribution.

- 'Location of new airport': two alternative future developments are examined in this case: a) with new airport and b) without new airport.

For every single future development of a hypothesis, a specific probability was assigned, based on expert knowledge. The above hypotheses, together with their probabilities, were used as input to the MORPHOL module (see Figure 5). Certain inclusion - exclusion constraints were also set by the research team, based on the knowledge gained so far for the study area, in order to decrease the number of possible scenarios to a manageable and meaningful total.

From all possible scenarios coming out of the MORPHOL module, a subset was selected on the basis of scenarios' probability. This was further evaluated / elaborated in terms of closeness and cohesiveness of scenarios, taking also into account the specific attributes of the region, results delivered from previous steps, stakeholders' view etc. In conclusion, two distinct scenarios are defined for the Kastelli municipality:

- S1: Scenario 'with new airport'; and

- S2: Scenario 'without new airport'.

Key aspect of both scenarios is technology. These have as follows:

A) Scenario 'with new airport'- S1

The first scenario considers the location of the new airport in the region of Kastelli, which takes away a considerable part of agricultural but also residential land. A new concentrated settlement pattern is emerging in the area, as a result of the population relocation to already existing or new residential settlements. As cultivated agricultural land is also significantly decreased, emphasis is placed on the role of technology and innovation as 'tools' towards the more effective use of the remaining agricultural land, together with agri-food technology for improving productivity of this land. The role of multifunctionality of the remaining agricultural land is also stressed, through the development of alternative tourism, processing of agricultural products (manufacturing), energy production from agri-waste management, etc. The role of ICTs is very important in this scenario, in serving the diffusion of knowledge and information relating to: technological advances relative to the agri-food sector, agricultural policies, cooperation opportunities, new agricultural markets, networking between firms, promotion of agri-food products etc. In addition, ICTs have a significant contribution to the development of other activities as well, e.g. tourist activities, trade sector, activities relating to social equity, etc. Moreover, the location of the airport and the improvement of the region's infrastructure networks (new or improved road transport network) contribute to the development of a range of activities in the study region, through the establishment of new or relocation of existing businesses from the whole Herakleion Prefecture. 


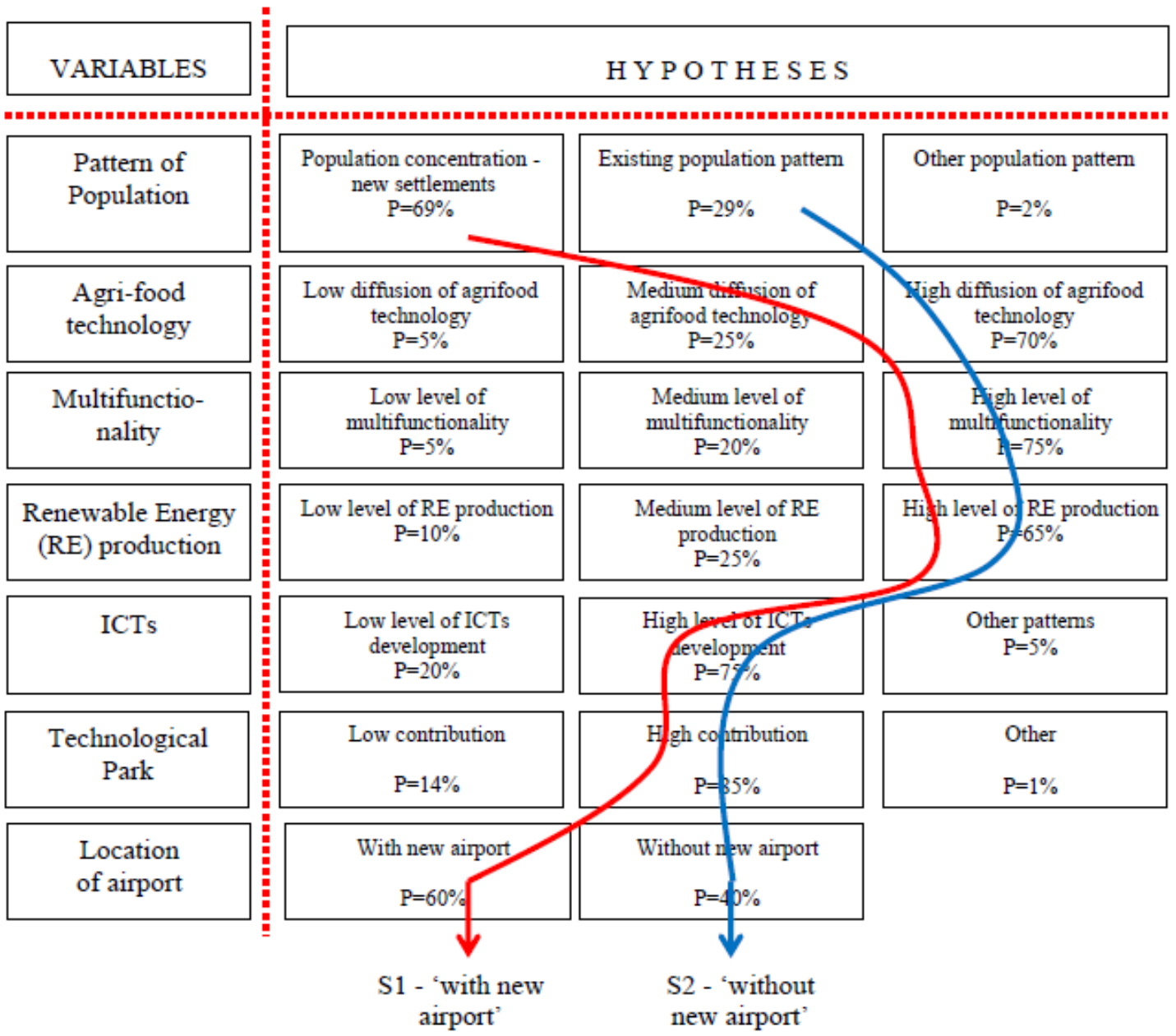

Figure 5. Structuring scenarios for the Kastelli region by use of the MORPHOL module

Source: Stratigea, Giaoutzi \& Papadopoulou, 2010

B) Scenario 'without new airport'- S2

This scenario does not include the location of the airport in the study area. Thus the existing spatial pattern of settlements remains unchanged, while the same holds for the population distribution pattern. Technology and innovation are considered as 'tools' towards the protection/preservation of natural resources of the Kastelli region. ICTs play a substantial role for networking among firms and also for marketing purposes. The construction of a more environmentally-responsible image of the region attracts tourist flows as well as small scale investments around eco-activities. Renewables gain ground as a mean to preserve local assets. Environmental culture prevails in the region, based on the increased awareness of the local community and the strong participation in the decision making processes. Agriculture in this scenario is considered not only as a production sector but as the core for safeguarding nature and developing small scale eco-entrepreneurship.

\subsection{Evaluation of Scenarios - The MULTIPOL Approach}

In the last step, the evaluation of scenarios in respect to the most proper policies and actions for each scenario implementation is carried out by use of the MULTIPOL multicriteria evaluation module.

\subsubsection{Data Input}

The information needed for feeding the MULTIPOL evaluation process involves the:

- scenarios, as defined in the previous step (MORPHOL module),

- pool of policies (strategic paths), supporting the implementation of scenarios,

- pool of actions (measures), serving different policies, and 
- evaluation criteria, emanating from the goal and objectives set in the planning problem at hand.

In the case of Kastelli region, these have as follows:

$\checkmark$ Scenarios

Two scenarios are used as input to the MULTIPOL module, being equally weighted:

- S1- with airport- Weight 5

- S2- without airport- Weight 5

$\checkmark$ Pool of policies

Policies, in the context of LIPSOR, are alternative strategic directions, driving the system at hand towards certain future developments. To each policy, a specific weight can be attached, reconciling political priorities, values and visions of local society and views of stakeholders, participating in the whole exercise. Policies structured for the Kastelli case study have as follows:

- Policy 1: 'Multifunctionality of agricultural land'. Rural development of the region can be based on the multifunctional role of agricultural land. Emphasis is placed on the agri-sector (organic agriculture), the alternative tourism (agrotourism, eco- tourism etc.), the production of biofuels (processing of agricultural waste and energy crops), and the processing of agri-food products. Technology is used as a tool for the sustainable development of the study region.

- Policy 2: 'Specialization in the agri-food sector'. Vertical structure of the production process (agricultural production - manufacturing - marketing - trading). Technology is used as a tool for increasing productivity and competitiveness, with emphasis on the quality and safety of agri-food products.

- Policy 3: 'Technological / training node'. The Kastelli region is developed as a technological and training node in agriculture in the greater region of the Herakleion Prefecture. This implies the: spatial concentration of technological and training services in the area; use of improved transport infrastructure networks (new airport Herakleion port - road network); close cooperation with research institutions and the University of Herakleion; and heavy use of the expanded telecommunication infrastructure (ICTs).

- Policy 4: 'Tourism and culture'. Rural development is based on the sustainable use of natural and cultural resources (ecosystems, archeological sites, traditional settlements, traditional products, etc.), rendering the region a 'cultural node' in the greater area.

$\checkmark$ Policy actions (measures)

Actions are measures that are serving a specific policy. The pool of actions considered in the Kastelli case study has as follows (Table 3):

Table 3. Policy actions (measures) for the Kastelli region

\begin{tabular}{ll}
\hline & \\
\hline A1 & Process modernization in the agricultural sector \\
A2 & Restructuring of agricultural production - Intensification pattern of agricultural land \\
A3 & Quality of agricultural products - Product labelling \\
A4 & Restructuring and upgrading of road infrastructure network \\
A5 & Training - Upgrading local skills and knowledge stock \\
A6 & Management - Trading of local agri-food production \\
A7 & Networking among firms (e.g. agricultural firms, tourist firms) \\
A8 & Development of agrotourism \\
A9 & Development of eco-tourist activities in environmentally sensitive parts of the Kastelli region \\
A10 & Development of walking paths - mountainous tourism \\
A11 & Energy production from biomass (agricultural waste's processing - energy crops) \\
A12 & Energy production (wind energy - solar energy) \\
A13 & ICTs - Development/upgrading of network infrastructure \\
A14 & Promotion of innovation with emphasis on the agri-food sector \\
A15 & Processing and promotion of agri-food products \\
A16 & Development of region's mountainous part \\
A17 & Settlement and cultural development \\
A18 & Development of a technological/training centre \\
\hline
\end{tabular}


Evaluation criteria

Evaluation criteria are emanating from the objectives set in the Kastelli case study, while they are prioritized (weights) with the help of local stakeholders (Table 4).

Table 4. Evaluation criteria in the Kastelli case study

\begin{tabular}{|c|c|c|c|c|}
\hline $\mathrm{a} / \mathrm{a}$ & Objectives & Criteria & & Weight* \\
\hline \multirow{4}{*}{1} & \multirow{4}{*}{ Environmental protection } & Noise pollution & K1 & 5 \\
\hline & & Biodiversity & K2 & 5 \\
\hline & & Landscape & $\mathrm{K} 3$ & 5 \\
\hline & & Water resources pollution & K4 & 6 \\
\hline 2 & Social cohesion & Employment & K5 & 5 \\
\hline \multirow{4}{*}{3} & \multirow{4}{*}{ Economic efficiency } & Primary sector & K6 & 3 \\
\hline & & Secondary sector & K7 & 3 \\
\hline & & Tertiary sector & K8 & 3 \\
\hline & & Competitiveness (long- term) & K9 & 3 \\
\hline \multirow{3}{*}{4} & \multirow{3}{*}{$\begin{array}{l}\text { Agricultural development } \\
\text { (multifunctionality) }\end{array}$} & Agricultural income & K10 & 3 \\
\hline & & Organic agriculture - livestock & K11 & 3 \\
\hline & & Energy crops & K12 & 3 \\
\hline 5 & Energy production & $\begin{array}{l}\text { Energy production from RES (Note 3) (wind parks, } \\
\text { biomass etc.) }\end{array}$ & K13 & 3 \\
\hline \multirow[t]{3}{*}{6} & Accessibility & Condition of road network & K14 & 4 \\
\hline & & Precise farming & K15 & 4 \\
\hline & & Product labelling & K16 & 4 \\
\hline \multirow[t]{3}{*}{7} & Innovation diffusion & Number of social enterprises (women partnerships) & K17 & 4 \\
\hline & & Number of new businesses & K18 & 4 \\
\hline & & New employment & K19 & 4 \\
\hline 8 & Preservation of cultural heritage & Number of cultural events & K20 & 5 \\
\hline
\end{tabular}

* Weights: range from 1 to 6 in ascending order of priority

$\checkmark$ Types of evaluation

MULTIPOL incorporates two different types of multicriteria evaluation, namely:

- The 'actions/policies evaluation' that evaluates actions (measures) in respect to policies, indicating which actions best fit to each single policy, thus providing an actions' hierarchy as to their impact on each policy.

- The 'policies/scenarios evaluation' that evaluates policies in respect to scenarios, indicating the policy which best fits to each specific scenario, thus providing a policies' hierarchy as to their impact on each scenario.

\subsubsection{Evaluation Results}

The results obtained by the two types of evaluation (action vs policy evaluation and policy vs scenario evaluation) are presented in the following.

$\checkmark$ Performance of actions in respect to policies

The performance of actions in respect to policies is presented in Table 5. The interpretation of these results is based on the combination of the mean value and standard deviation scores of actions, where: the mean value measures the performance of each specific action for each policy; while the standard deviation characterizes the actions' sensitivity in respect to policies. The higher the mean value and the lower the standard deviation of an action as to a certain policy is, the better its performance. Moreover, actions with low standard deviation may fit well to more than one policy; while, actions with high standard deviation fit well to specific policies, provided that they have a good performance score in respect to the policies considered. 
Table 5. Evaluation of actions in respect to policies

\begin{tabular}{cccccccc}
\hline & P1 & P2 & P3 & P4 & $\begin{array}{c}\text { Mean } \\
\text { value }\end{array}$ & $\begin{array}{c}\text { Standard } \\
\text { deviation }\end{array}$ & Position* \\
\hline A1 & 10.2 & 10.9 & 10.3 & 9.8 & 10.2 & 0.4 & 4 \\
A2 & 9.4 & 9.9 & 10.1 & 9.4 & 9.6 & 0.3 & 3 \\
A3 & 11.1 & 11.7 & 11.2 & 10.8 & 11.1 & 0.3 & 9 \\
A4 & 9.2 & 8.7 & 9.4 & 9.9 & 9.3 & 0.4 & 17 \\
A5 & 13.3 & 13.6 & 13.8 & 13.2 & 13.4 & 0.2 & 13 \\
A6 & 12.4 & 12.7 & 12.1 & 12.3 & 12.4 & 0.2 & 18 \\
A7 & 11 & 11.5 & 11 & 10.6 & 11 & 0.3 & 7 \\
A8 & 14.5 & 14.1 & 14 & 14.4 & 14.3 & 0.2 & 5 \\
A9 & 11 & 9.6 & 11 & 11.5 & 10.8 & 0.6 & 1 \\
A10 & 10.5 & 9.4 & 10.4 & 10.9 & 10.4 & 0.5 & 15 \\
A11 & 11.6 & 11.3 & 12.2 & 11.5 & 11.6 & 0.3 & 12 \\
A12 & 8.7 & 8.5 & 9.3 & 8.9 & 8.9 & 0.3 & 10 \\
A13 & 13.3 & 13.6 & 12.7 & 12.8 & 13.1 & 0.4 & 6 \\
A14 & 12 & 12.6 & 11.9 & 11.9 & 12.1 & 0.3 & 16 \\
A15 & 13.1 & 13.5 & 12.9 & 12.7 & 13 & 0.3 & 0.3 \\
A16 & 11.3 & 10.8 & 10.9 & 11.6 & 11.2 & 0.4 & 0.2 \\
A17 & 11 & 10.1 & 10.5 & 11.3 & 10.8 & 13.2 & 0.3 \\
A18 & 13 & 13.1 & 13.5 & 13.2 & 13.2 & \\
\hline
\end{tabular}

* Hierarchy of actions, based on their performance as to the policies considered (in ascending order)

Actions with higher performance

Actions with lower performance

Source: Stratigea, Giaoutzi \& Papadopoulou, 2010

For the Kastelli case study, the actions exhibiting high performance and low standard deviation scores, fitting thus well to more than one policy are (Table 5): A5 (Training - Upgrading local skills and knowledge stock); A6 (Management Trading of local agri-food production); A8 (Development of agrotourism); A18 (Development of technological/training centre); A15 (Processing and promotion of agri-food products); A11 (Energy production from biomass); A14 (Promotion of innovation with emphasis on the agri-food sector); and A13 (ICTs Development/upgrading of network infrastructure). Such types of actions (measures) are of importance, as they increase flexibility and reduce risks of policy making, associated with unexpected changes of the internal and external environment (e.g. changing food consumption patterns or technological changes) that may call for a shift in policy direction.

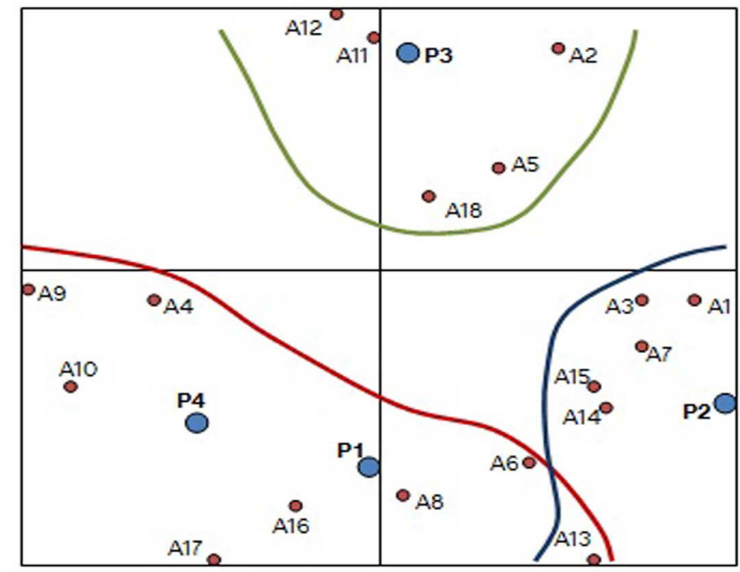

Figure 6. Actions/ Policies closeness map

Source: Stratigea, Giaoutzi \& Papadopoulou, 2010

\section{Policy Packages}

The actions/policies closeness map (Figure 6) indicates those measures that fit best to each of the policies considered. 
In this way, 'policy packages' are constructed for each policy. It also indicates that Policy 1 (multifunctionality) and Policy 4 (tourism-culture) are close policies, mostly served by the same package of measures (see also Table 5).The following policy packages are created for each policy (Tables 6 to 8):

Table 6. Policy package for Policy 1 and Policy 4

\begin{tabular}{cl}
\hline Policy & \multicolumn{1}{c}{ Policy Package (Set of Policy Measures) } \\
\hline Policy 1 & A4: Restructuring and upgrading of road infrastructure network \\
Multifunctionality & A6: Management - Trading of local agri-food production \\
& A8: Development of agrotourism \\
and & A9: Development of eco-tourist activities in environmentally sensitive parts \\
& A10: Development of walking paths - mountainous tourism \\
Policy 4 & A13: ICTs - Development/upgrading of network infrastructure \\
Tourism and Culture & A16: Development of the mountainous compartments of the study region \\
& A17: Settlements and cultural development \\
\hline
\end{tabular}

Table 7. Policy package for Policy 2

\begin{tabular}{cl}
\hline Policy & \multicolumn{1}{c}{ Policy Package (Set of Policy Measures) } \\
\hline & A1: Process modernization in the agricultural sector \\
Policy 2 & A3: Quality of agricultural products - Product labelling \\
& A7: Networking among firms (e.g. agricultural firms, tourist firms) \\
Specialization in the food sector & A13: ICTs - Development/upgrading of network infrastructure \\
& A14: Promotion of innovation with emphasis on the agri-food sector \\
& A15: Processing and promotion of agri-food products \\
\hline
\end{tabular}

Table 8. Policy package for Policy 3

\begin{tabular}{cl}
\hline Policy & \multicolumn{1}{c}{ Policy Package (Set of Policy Measures) } \\
\hline & A2: Restructuring of agricultural production - Intensification pattern of agricultural land \\
Policy 3 & A5: Training - Upgrading of local skills and knowledge stock \\
& A11: Energy production from biomass (processing of agricultural waste - energy crops) \\
$\begin{array}{c}\text { Technological and } \\
\text { training node }\end{array}$ & A12: Energy production (wind - solar energy) \\
$\checkmark$ & A18: Development of a technological/training centre \\
\hline
\end{tabular}

$\checkmark$ Performance of policies in respect to scenarios

In Table 9, the performance of policies P1 (multifunctionality), P2 (specialization in the agri-food sector), P3 (technological/training node) and P4 (tourism/culture) in respect to the two scenarios is presented, while mean value and standard deviation of their performance is depicted in Figure 7.

Table 9. Scores of policies in respect to scenarios

\begin{tabular}{cccccc}
\hline & S1 & S2 & $\begin{array}{c}\text { Mean } \\
\text { value }\end{array}$ & $\begin{array}{c}\text { Standard } \\
\text { deviation }\end{array}$ & Position \\
\hline P1 & 5.3 & 4.9 & 5.1 & 0.2 & 3 \\
P2 & 5.2 & 4.9 & 5 & 0.1 & 2 \\
P3 & 5 & 5 & 5 & 0.0 & 1 \\
P4 & 5.3 & 4.9 & 5.1 & 0.2 & 4 \\
\hline
\end{tabular}

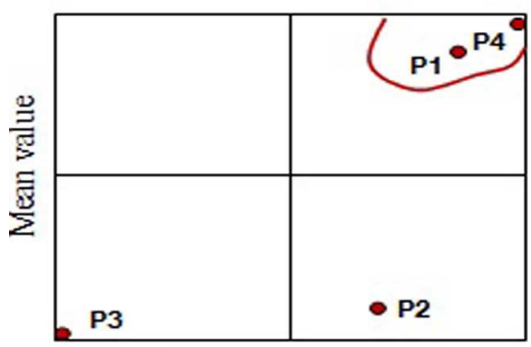

Standard deviation

Figure 7. Classification of policies

Source: Stratigea, Giaoutzi \& Papadopoulou, 2010 


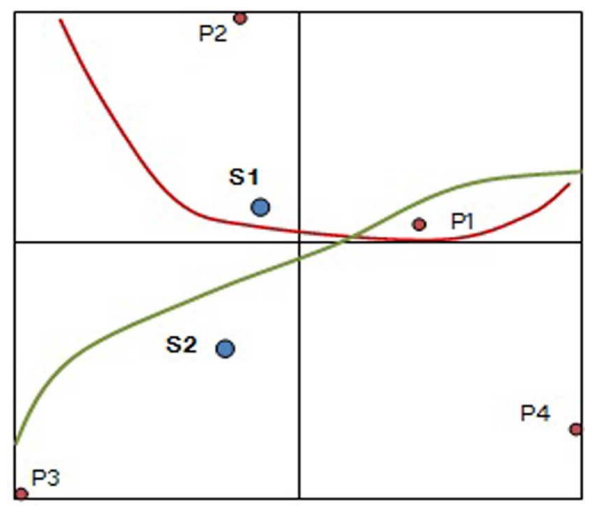

Figure 8. Policies/ scenarios closeness map

Source: Stratigea, Giaoutzi \& Papadopoulou, 2010

In Figure 8, the closeness of policies in respect to scenarios S1 ('with new airport') and S2 ('without new airport') is presented, providing an indication of the strategic direction to be followed by the Kastelli region in each specific scenario in order to reach the objectives set.

More precisely:

Scenario S1 ('with new airport') is better served by:

- $\quad$ P1 (multifunctionality) and

- $\quad$ P2 (specialization in the food sector)

The location of the new airport in the Kastelli region will have a strong influence on the loss of a considerable part of agricultural land (the Kastelli plain). As a compromise to that loss, the region pursues the increase of multifunctionality of the remaining land through the development of complementary activities (agricultural, manufacturing, alternative tourism activities, etc.), in order to support local income. At the same time, by taking advantage of technological developments, the region places emphasis on the specialization in the agri-food sector, focusing on qualitative agri-food production.

Scenario S2 ('without new airport') is better served by:

- $\quad$ P4 (tourism - culture);

- $\quad$ P1 (multifunctionality); and

- $\quad$ P3 (technological/ educational node)

In the second scenario, rural development is based on the multifunctionality of agricultural land (agricultural sector, manufacturing, alternative tourism etc.). Emphasis is also placed on: the sustainable development of natural and cultural resources, based on the adoption-use of technological innovation; and the development of the region as a technological and training centre, which will contribute to the upgrading of existing skills in the local population and the increase of knowledge stock available in the area.

\section{Conclusions}

In the present paper, the emphasis is on the structuring of a participatory framework for foresighting future development of a specific rural region (regional level). This framework attempts to integrate a range of participatory tools, with placing the LIPSOR participatory model at the heart of the framework, while complementing this with the Focus Groups methodology and the Future Workshop participatory approach. The last two are mainly used for structuring discussions, taking place in participatory workshops at the municipality of Kastelli. In these workshops, exchange of information and experience has taken place between researchers and decision makers, on the one hand; and public and local stakeholders and experts, on the other.

The experience gained from the application of this participatory framework stresses the value added by the participation of stakeholders, experts and local administrative agencies in the whole planning exercise. This is largely relating to the: increased effectiveness of the planning efforts (process, content and outcome), by refining the perception of professional planners on local issues and their sense of the local 'feeling'; 'trust' developed among all partners involved in this planning exercise; better understanding of different views and reduction of conflicts among 
local groups; consensus building on policies and measures relevant to cope with future challenges involved by the potential location of the new airport, etc. The proposed policy framework, as the final outcome of this effort, is of value as it represents a co-design effort of planners and the local community. This can support the Kastelli region in coping with challenges and opportunities of the rapidly changing internal and external environment.

\section{References}

Barbanente, A., Khakee, A., \& Puglisi, M. (2002). Scenario Building for Metropolitan Tunis. Futures, 34, 583-596. http://dx.doi.org/10.1016/S0016-3287(02)00002-2

Berg, B. L. (1989). Qualitative Research Methods for the Social Sciences. Allyn and Bacon, Boston.

Dürrenberg, J. Behringer, U. Dahinden, A. Gerger, B. Kasemir, C. Querol, R., ... C. Jaeger. (1997). Focus Groups in Integrated Assessment - A Manual for a Participatory Tool. ULYSSES Working Paper WP-97-92. Darmstadt, Germany.

Godet, M. (1994). From Anticipation to Action: A Handbook of Strategic Perspective. UNESCO Publishing.

Godet, M. (1999). Creating the Future. The Antidote, (22), 11-16.

Godet, M. (2001). Creating Futures: Scenario Planning as a Strategic Management Tool. Economica.

Godet, M., R. Monti, F. Meunier, \& F. Roubelat. (2004). Scenarios and Strategies: A Toolbox for Problem Solving. Cahiers du LIPSOR, Laboratory for Investigation in Prospective and Strategy, Paris.

Jungk, R., \& Mullert, N. (1987). Future Workshops: How to Create Desirable Futures. London, Institute for Social Inventions.

Kasemir, B., Dahinden, U., Gerger, Å., Schüle, R., Tabara, D., \& Jaeger, C.C. (2000). Citizens' Perspectives on Climate Change and Energy Use. Global Environmental Change, 3, 169-184. http://dx.doi.org/10.1016/S0959-3780(00)00022-4

Organisation for Economic Co-operation and Development [OECD]. (2006). The new rural paradigm - policies and governance. OECD Publishing.

Rotmans, J. (1998). Methods for IA: The Challenges and Opportunities Ahead. Environmental Modelling and Assessment, 3, 155-179. http://dx.doi.org/10.1023/A:1019019024003

Stewart, D. W., \& Shamdasani, P. N. (1990). Focus Groups: Theory and Practice. Sage Publications, Newbury Park, California.

Stratigea, A., Giaoutzi, M., \& Papadopoulou, Ch.-A. (2010). Foresight Analysis in AG2020: The Case of Kastelli Herakleion-Crete - A Participatory Methodological Framework. Deliverables 6.1, 6.2, 6.3 and 6.4, AG2020 Project.

Stratigea, A. (2011). ICTs for Rural Development: Potential Applications and Barriers Involved. NETCOM, 25(3-4), 179-204.

Stratigea, A., Grammatikogiannis, E., \& Giaoutzi, M. (2012). How to Approach Narratives in Foresight Studies: Qualitative Data Analysis. In E. van Leeuwen, M. Giaoutzi and P. Nijkamp (Eds.), Future Perspectives for Sustainable Agriculture. International Journal of Foresight and Innovation Policy (IJFIP), Special Issue, 8(2/3), 236-261. http://dx.doi.org/10.1504/IJFIP.2012.046112

\section{Notes}

Note 1. 'Simple' probability: the occasion that a hypothesis will be materialized in a predefined time horizon.

Note 2. 'Conditional' probabilities: a) Pi/j: the occasion that a hypothesis i will be materialized, if another hypothesis $\mathrm{j}$ has been materialized before; and b) Pi/nonj: the occasion that a hypothesis $\mathrm{i}$ will be materialized, if another hypothesis $\mathrm{j}$ has not been materialized before.

Note 3. RES: Renewable Energy Sources 